\title{
SUPLEMENTAÇÃO E ALIMENTAÇÃO ADEQUADA NO CONTEXTO ATUAL DA PANDEMIA CAUSADA PELA COVID-19
}

Supplementation and proper nutrition in the current pandemic context caused by covid-19

Suplementación y alimentación correcta en el contexto pandémico actual causado por covid-19

Bárbara Paixão de Gois ${ }^{1 *}$, Araída Dias Pereira² ${ }^{2}$ Karem Lays Soares Lopes ${ }^{1}$, Flávia Campos Corgosinho ${ }^{3}$

${ }^{1}$ Mestranda no Programa de Pós-graduação em Nutrição e Saúde da Universidade Federal de Goiás, Grupo de Pesquisa de Estudos da Obesidade, Goiânia, Brasil.

${ }^{2}$ Profa. Dra. do Curso de Nutrição e Laboratório de Pesquisa Clínica da Universidade Federal do Tocantins, Palmas, Brasil.

${ }^{3}$ Profa. Dra. do Curso de Nutrição e Programa de Pós-Graduação em Nutrição e Saúde da Universidade Federal de Goiás, Grupo de Pesquisa de Estudos da Obesidade, Goiânia, Brasil.

*Correspondência: Grupo de Pesquisa da Obesidade, Universidade Federal de Goiás - UFG, Programa de Pós-Graduação em Nutrição e Saúde, Rua 227 Qd. 68 s/no - Setor Leste Universitário, Goiânia, Goiás, Brasil. CEP: 74.605-080 e-mai: babipaixao@uft.edu.br

\section{Artigo recebido em 03/04/2020 aprovado em 21/04/2020 publicado em 22/04/2020}

\section{RESUMO}

O surto do coronavírus foi classificado pela Organização Mundial da Saúde, como pandemia. Desde então, o assunto vem sendo abordado de forma exaustiva nas redes sociais e veículos de comunicação em massa. A importância de uma alimentação balanceada e rica em nutrientes, além de enfatizar que não existem superalimentos, fórmulas, "shots", sucos ou soroterapias por infusão endovenosa de nutrientes, que sejam indicados para prevenir ou até mesmo tratar pessoas contaminadas pelo coronavírus, precisa ser reforçada. Esse artigo tem como objetivo informar os profissionais da saúde e população em geral, acerca do papel da alimentação e da real necessidade do uso de suplementos alimentares nesse contexto. As informações utilizadas basearam-se nos conhecimentos já consolidados na literatura sobre nutrição, e em documentos científicos e oficiais até então divulgados. Alguns nutrientes como Vitamina A, D, C, Complexo B, Ferro, Zinco e Selênio podem atuar de maneira positiva no sistema imunológico, no entanto, uma alimentação balanceada é capaz de fornecer esses nutrientes. Já o uso de suplementação para melhora da imunidade na prevenção e tratamento da COVID-19 não tem respaldo cientifico até o momento.

Palavras-chave: COVID-19, Alimentação saudável, Imunidade.

\section{ABSTRACT}

The coronavirus outbreak was classified by the World Health Organization as a pandemic. Since then, the subject has been discussed extensively in social networks and mass communication vehicles. The importance of a balanced and nutrient-rich diet, in addition to emphasizing that there are no superfoods, formulas, shots, juices or serotherapies by intravenous infusion of nutrients, which are indicated to prevent or even treat people infected with the coronavirus, needs to be considered. This article aims to inform health professionals and the population in general, about the role of food and the real need to use dietary supplements in this context. All the information was based on the knowledge already consolidated 
in the literature on nutrition, and on scientific and official documents published until now. Some nutrients such as Vitamin A, D, C, Complex B, Iron, Zinc and Selenium can act positively on the immune system, however, a balanced diet is able to provide these nutrients. The use of supplementation to improve immunity in the prevention and treatment of COVID-19 has not been scientifically supported yet.

Keywords: COVID-19, Healthy eating, Immunity.

\section{RESUMEN}

El brote de coronavirus fue clasificado por la Organización Mundial de la Salud como una pandemia. Desde entonces, el tema se ha debatido ampliamente en las redes sociales y los vehículos de comunicación de masas. Es necesario tener en cuenta la importancia de una dieta equilibrada rica en nutrientes, además de enfatizar que no hay superalimentos, fórmulas, inyecciones, jugos o seroterapias por infusión intravenosa de nutrientes, que están indicados para prevenir o incluso tratar a las personas infectadas por el coronavirus. Este artículo tiene como objetivo informar a los profesionales de la salud y a la población en general sobre el papel de los alimentos y la necesidad real de usar suplementos dietéticos en este contexto. La información utilizada se basó en el conocimiento ya consolidado en la literatura sobre nutrición y en documentos científicos y oficiales publicados previamente. Algunos nutrientes como la vitamina $A, D, C$, complejo $B$, hierro, zinc y selenio pueden actuar positivamente sobre el sistema inmunitario, sin embargo, una dieta equilibrada puede proporcionar estos nutrientes. El uso de suplementos para mejorar la inmunidad en la prevención y el tratamiento de COVID-19 aún no ha sido respaldado científicamente.

Descriptores: COVID-19, Alimentación saludable, Inmunidad.

\section{INTRODUÇÃO}

O surto do coronavírus (SARS-CoV-2) foi classificado pela Organização Mundial da Saúde (OMS), como pandemia. Desde então, o assunto vem sendo abordado de forma exaustiva nas redes sociais e veículos de comunicação em massa. No que diz respeito a nutrição, as informações que objetivam orientar a população sobre o consumo de suplementos e alimentos no combate ou prevenção da doença, tem ganhado muita repercussão (CFN, 2020).

Em nota oficial em sua página, o Conselho Federal de Nutricionistas alertou a população e os profissionais da área a não acreditarem em notícias divulgadas em meios não oficiais. Reforçou também, a importância de uma alimentação balanceada e rica em nutrientes, além de enfatizar que não existem superalimentos, fórmulas, "shots", sucos ou soroterapias por infusão endovenosa de nutrientes, que sejam indicados para prevenir ou até mesmo tratar pessoas contaminadas pelo vírus (CFN, 2020).

Em 20 de março de 2020, foi declarado pelo Ministério da Saúde a transmissão comunitária da COVID-19 em todo o Brasil. A partir desse dia, a preocupação em conter a disseminação do vírus e em proteger especialmente os grupos de risco foi acentuada, e as campanhas de conscientização intensificadas. Os últimos dados divulgados pela Organização Pan-Americana da Saúde (OPAS, 2020), mostraram que cerca de $81 \%$ dos casos da COVID-19 progridem para leve ou moderado, $14 \%$ para grave e apenas $5 \%$ para crítico, sendo que os idosos, pessoas com Doenças Crônicas não Transmissíveis (DCNTs), doenças pulmonares e câncer, também, parecem desenvolver doenças graves mais frequentemente 
que os outros (ZHOU et al., 2020; WANG et al., 2020; OPAS, 2020).

A melhor forma de prevenir o contágio é a quarentena, quando possível. Também é muito importante a higienização das mãos, que se cubra o nariz e a boca ao espirrar ou tossir, que se evite aglomerações, que os ambientes estejam sempre ventilados e que os objetos pessoais não sejam compartilhados. Não há qualquer comprovação cientifica a respeito do uso de alimentos ou suplementes alimentares para prevenção (BRASIL, 2020; WATKINS, 2020).

Por se tratar de um tipo de coronavírus, as pesquisas tem sido direcionadas com base nas experiências com a Síndrome Respiratória Aguda (SARS) e a Síndrome Respiratória do Oriente Médio (MERS) que também são causadas por outros tipos de coronavírus, para tentar entender como o sistema imunológico responde ao mesmo, já que podem se passar meses ou até mesmo anos, até que se consiga decifrar todas as nuances do recém descoberto SARS-CoV-2, causador da COVID-19 (PROMPETCHARA; KETLOY; PALAGA, 2020).

Nesse sentido, esse artigo tem como objetivo informar os profissionais da saúde e população em geral, a cerca do papel da alimentação e da real necessidade do uso de suplementos alimentares, no contexto atual da COVID-19. As informações aqui disponibilizadas baseiam-se nos conhecimentos já consolidados na literatura sobre nutrição, e em alguns documentos científicos e oficiais divulgados, desde o início da pandemia pelos órgãos governamentais e revistas científicas.

\section{BENEFÍCIOS DA ALIMENTAÇÃO EQUILIBRADA}

Nutrir-se vai muito além da prática de satisfazer a fome. A nutrição é um componente crucial do desenvolvimento e da saúde humana. Sabe-se que uma alimentação equilibrada e saudável está relacionada, dentre outras coisas, a concepção, a gestação, a recuperação no puerpério e ao aleitamento materno apropriado. Além disso, está envolvida com o crescimento adequado na infância, melhor resposta imune, menor risco de desenvolvimento de doenças, e a uma expectativa de vida maior. Deste modo, uma dieta saudável e equilibrada é capaz de promover a saúde, bem como prevenir doenças (BARTRINA et al., 2006; WHO, 2018).

A alimentação deve ser variada e composta por alimentos de boa qualidade nutricional. A quantidade deve ser adequada, uma vez que tanto a sub quanto a hiper nutrição, são igualmente prejudiciais. No que diz respeito à população brasileira, é recomendado que a dieta seja composta em sua maioria, por alimentos in natura ou minimamente processados (ABARCAGÓMEZ et al., 2017; BRASIL, 2014).

Os processados devem ser consumidos de maneira limitada, e os ultraprocessados sempre que possível, evitados. Recomenda-se ainda, que o consumo de sal, açucares e gorduras seja feito em pequenas quantidades, sendo que estes estão intimamente relacionados com o surgimento de doenças, se o uso for indiscriminado. Quando tais 
recomendações são praticadas, o organismo mantém boas condições de saúde (BRASIL, 2014; ABARCA-GÓMEZ et al., 2017; WHO, 2018).

Em contra partida, uma alimentação desequilibrada, quanti e qualitativamente pode gerar uma série de consequências. Há muito é consolidado na ciência que a alimentação é capaz de impulsionar ou reduzir as chances do desenvolvimento de doenças, especialmente das Doenças Crônicas Não Transmissíveis (DCNTs). As DCNTs são um grupo de enfermidades que incluem obesidade, doenças cardiovasculares, diabetes, hipertensão arterial, câncer e algumas enfermidades pulmonares, estas diminuem a qualidade e expectativa de vida (ABARCAGÓMEZ et al., 2017).

O desenvolvimento e agravo de DCNTs está diretamente relacionado a hábitos alimentares ruins e seu tratamento inclui a mudança de comportamento. Destaca-se ainda, o fato de que pacientes portadores de DCNTs estão mais suscetíveis a ação de doenças oportunistas, como algumas doenças virais e bacterianas. Neste sentido, essas pessoas compõem o grupo de risco de indivíduos que estão mais vulneráveis à ação de doenças como a COVID-19 (GASMI, et al., 2020; CDC, 2020).

Nesta perspectiva, é de suma importância ressaltar que a alimentação tem papel fundamental na condição de saúde dos indivíduos, sendo capaz de potencializar a ação do sistema imune. Deve-se destacar ainda, que a alimentação saudável se baseia na diversidade de consumo de alimentos de boa qualidade e em quantidades adequadas, e deve ser praticada continuamente para potencializar seus benefícios, não sendo a alimentação por si só uma garantia de que indivíduos se tornem imunes a COVID-19 (GASMI, et al., 2020).

\section{ALIMENTAÇÃO E IMUNIDADE}

Muitos são os fatores que podem influenciar na nossa imunidade como sono, atividade física, fatores emocionais e a alimentação (LASSELIN; ALVAREZ-SALAS; GRIGOLEIT, 2016).

A energia e os nutrientes obtidos por meio dos alimentos desempenham um papel importante no desenvolvimento e preservação do sistema imunológico, portanto, qualquer desequilíbrio nutricional afeta sua competência e integridade (LÓPEZ; BERMEJO, 2017). Entretanto, não existe um superalimento ou fórmula nutricional, com comprovação científica, capaz de impedir as contaminações virais (CFN, 2020).

Alguns nutrientes como Vitamina A, C, Ferro, Zinco e Selênio podem atuar de maneira positiva no sistema imunológico. Em condições fisiológicas normais, é possível atingir as necessidades diárias desses micronutrientes (MAGGINI et al., 2018; CFN, 2020).

A vitamina A ajuda a manter a integridade estrutural e funcional das células da pele, do trato respiratório e outros. Além disso, é importante para o bom funcionamento das células do sistema imune. Alimentos de origem animal (retinol) e vegetais alaranjados e verde-escuros (pró- 
vitamina A) são ricos na mesma (Tabela 1) (MAGGINI et al., 2018).

A vitamina $\mathrm{C}$, dentre as suas diversas funções, atua como um importante antioxidante, pode aumentar o número de anticorpos e age na diferenciação e proliferação de células do sistema imune (CARR; MAGGINI, 2017). As frutas cítricas são as principais fontes dessa vitamina (Tabela 1).

Dentre as várias funções do ferro, ele é essencial para diferenciação e crescimento celular e componente de enzimas críticas para o funcionamento das células imunes (MAGGINI et al., 2018). Os alimentos de origem animal (ferro heme e não-heme) e de origem vegetal (ferro não heme) são fontes de ferro (Tabela 1).

O zinco é um importante antioxidante e exerce um papel central no crescimento celular e diferenciação de células imunes que apresentam rápida diferenciação e renovação (MAGGINI et al., 2018). O zinco pode ser encontrado em alimentos de origem animal, nas castanhas e sementes (Tabela 1).

O selênio atua auxiliando na regulação do sistema imunológico (MAGGINI et al., 2018). A principal fonte de selênio é a castanha-do-Brasil, apenas 1 unidade é capaz de fornecer $100 \%$ das recomendações diárias desse micronutriente (Tabela 1).

\section{SUPLEMENTAÇÃO, QUANDO É NECESSÁRIA?}

Os suplementos alimentares englobam plantas e suas partes, vitaminas, minerais, aminoácidos e diversos ingredientes que podem conter princípios bioativos essenciais ou não, que trazem benefícios a saúde.

Tabela 1. Principais alimentos ricos em vitaminas e minerais que auxiliam o sistema imunológico.

\begin{tabular}{c|l}
\hline MICRONUTRIENTE: & \multicolumn{1}{c}{ ALIMENTOS FONTE: } \\
\hline Vitamina A & $\begin{array}{l}\text { Pode ser encontrada em vários alimentos tanto de origem animal como } \\
\text { vegetal: ovos, leite, queijos, fígado, legumes e verduras de cor } \\
\text { alaranjada (abóbora, buriti, mamão, manga, cenoura) e de cor verde- } \\
\text { escuros (almeirão, agrião, couve, espinafre, ora-pro-nobis, rúcula). } \\
\text { As principais fontes de vitamina C são os alimentos cítricos como a } \\
\text { laranja, limão, mexerica, acerola, dentre outras. O mamão a couve e o } \\
\text { pimentão também são fontes de vitamina C. } \\
\text { Pode ser encontrado em alimentos de origem animal e vegetal: carnes } \\
\text { vermelhas, frango, feijão, guariroba, gergelim, jenipapo, mangaba, } \\
\text { mostarda, ora-pro-nobis, rúcula, taioba dentre outras. A ingestão de } \\
\text { alimentos fontes de vitamina C concomitante com fontes de ferro não- } \\
\text { heme, favorecem sua absorção. } \\
\text { É encontrado em alimentos de origem animal como carnes, peixes } \\
\text { (sardinha), ovos e em alguns alimentos de origem vegetal como: feijão, } \\
\text { lentilha, castanhas, gergelim e linhaça. } \\
\text { São fontes desse nutriente: castanha-do-Brasil, feijão, farinha de trigo } \\
\text { (integral), fubá de milho, macarrão integral e frutas como ameixa, } \\
\text { manga, maracujá e melancia, com destaque para a castanha-do-Brasil } \\
\text { que fornece uma generosa quantidade desse nutriente. }\end{array}$ \\
\hline
\end{tabular}

Fonte: Tabela brasileira de composição de alimentos-TACO. 2011; BRASIL, 2020.

Entretanto, é preciso entender que eles não agem como pílulas mágicas, neutralizando maus hábitos de vida e melhorando instantaneamente a saúde de quem os consome. 
É necessário que se tenha um estilo de vida saudável, com uma dieta balanceada, prática de atividade física e bons hábitos relacionados a saúde como um todo (KHAN et al., 2020).

Com objetivo de melhorar a imunidade, e consequentemente, minimizar os efeitos do coronavírus no organismo, a ideia de fazer uso de suplementos alimentares vem sendo amplamente divulgada nas redes sociais e veículos de massa, em sua maioria por pessoas leigas e sem nenhum embasamento científico (CFN, 2020).

De fato, é sabido que com o avançar da idade, o sistema imunológico passa por várias mudanças, e que algumas substâncias, como os microrganismos vivos conhecidos como probióticos, podem ser utilizados para melhorar a imunidade. Finamore et al. (2019), em um estudo randomizado, duplo-cego, controlado por placebo, suplementaram um grupo de 98 adultos com mais de 70 anos por 30 dias, com uma mistura de duas cepas de probióticos. Os resultados mostraram que a suplementação foi capaz de melhorar a resposta imune inata e adaptativa dos idosos suplementados.

Além disso, já é consenso que a ingestão de alimentos e ou suplementação que contém probióticos, prebióticos e simbióticos favorecem a preservação da microbiota presente no trato gastrointestinal e essa, exerce um papel importante agindo com barreira fisiológica. A colonização de microrganismos benéficos a saúde no intestino auxilia a resposta imune, sendo a alimentação equilibrada a melhor forma de fazer essa colonização, e assim, prevenir doenças. Produtos lácteos, iogurtes e lácteos fermentados são boas fontes de probióticos, já os prebióticos podem ser encontrados em frutas, legumes, condimentos e verduras, como banana, cebola, alho, chicória, tomate, dentre outros (PERBELIN et al., 2019).

Para fins de tratamento de pessoas já acometidas pela COVID-19 e que estão sob cuidados de profissionais da saúde como médicos e nutricionistas, um manejo nutricional com a tentativa de fortalecer o sistema imune e, ajudar o corpo a combater a ação do vírus, pode ser adotado. Com base na experiência adquirida com os coronavírus causadores da Síndrome Respiratória Aguda (SARS) e a Síndrome Respiratória do Oriente Médio (MERS), é possível realizar abordagem similar ao tratar a COVID-19 (ZHANG; LIU, 2020).

Os micronutrientes exercem papéis vitais no sistema imunológico, sendo os mais necessários para sustentar a imunocompetência, as vitaminas $\mathrm{A}, \mathrm{C}, \mathrm{D}$, complexo $\mathrm{B}$, betacaroteno, ferro, selênio e zinco. A resposta imune fica comprometida quando o estado nutricional se encontra inadequado, predispondo os indivíduos a infecções (MAGGINI; PIERRE; CALDER, 2018).

Além disso, o comprometimento nutricional pode ser exacerbado pela própria resposta imune a uma infecção. Assim, nesse momento delicado para a saúde pública, deve se ter uma atenção especial as necessidades diárias desses micronutrientes, que podem não ser alcançadas por meio da alimentação, e então, a suplementação torna-se uma alternativa 
(HARYANTO, et al. 2015; MAGGINI; PIERRE; CALDER, 2018).

A tabela 2 apresenta opções de suplementos nutricionais a serem adotados por nutricionistas ou médicos no suporte ao tratamento de pessoas hospitalizadas e acometidas pela COVID-19, com base na sua ação benéfica no auxílio do tratamento de doenças correlatas

Tabela 2. Opção de suplementação no auxílio ao tratamento da COVID-19.

\begin{tabular}{c|l}
\hline OPÇÃO & \multicolumn{1}{|c}{ VÍRUS ALVO E FUNÇÕES RELACIONADAS } \\
\hline Vitamina A & Vírus do sarampo, vírus da imunodeficiência humana, coronavírus aviário. \\
Complexo B & MERS-CoV; lesão pulmonar induzida por ventilador. \\
Vitamina C & Coronavírus aviário; infecções do trato respiratório inferior. \\
Vitamina D & Coronavírus bovino \\
Vitamina E & Coxsackievírus, coronavírus bovino. \\
Ômega - 3 & Vírus da gripe, vírus da imunodeficiência humana. \\
Selênio & Vírus da gripe, coronavírus aviário; mutações virais. \\
Zinco & Vírus do sarampo, SARS - CoV. \\
Ferro & Mutações virais. \\
\hline
\end{tabular}

Fonte: Adaptado de ZHANG e LIU, 2020.

É importante ressaltar, que a decisão de suplementar ou não, doses e tempo de suplementação, cabe a cada profissional, com base na história clínica do paciente e as recomendações para cada suplemento. A suplementação de indivíduos saudáveis sem orientação de um profissional não se justifica.

\section{CONSIDERAÇÕES FINAIS}

Dado o atual cenário onde a pandemia da COVID-19 assola o mundo, a propagação de informações relacionadas ao tema se dá de forma muito rápida e contínua. No que diz respeito a alimentação e nutrição, é preciso ter cuidado com orientações divulgadas por meios não oficiais e sem qualquer respaldo científico. Apesar do uso de suplementação para melhora da imunidade e estado nutricional de pacientes, estar bem consolidada na literatura, pouco se sabe sobre seu real benefício no auxílio ao tratamento da COVID-19, e até o momento, não há qualquer respaldo cientifico para essa prática ser adotada por indivíduos saudáveis ou que não apresentem alguma deficiência pré existente. É preciso ter cautela, e uma alimentação equilibrada ainda é a melhor forma de manter a saúde e prevenir doenças.

\section{REFERÊNCIAS}

ABARCA-GÓMEZ, Leandra et al. Worldwide trends in body-mass index, underweight, overweight, and obesity from 1975 to 2016: a pooled analysis of 2416 population-based measurement studies in 128. 9 million children, adolescents, and adults. The Lancet, v. 390, n. 10113, p. 2627-2642, 2017.

BARTRINA, J. A; SERRA-MAJEM, L; PEREZRODRIGO, C; RIBAS-BARBA, L; DELGADORUBIO, A. Nutrition risk in the child and adolescent population of the Basque country: the enKid Study. British journal of nutrition, v. 96, n. S1, p. S58-S66, 2006.

BRASIL. Ministério da Saúde. Guia Alimentar Para a População Brasileira. Brasília, DF. 2014. 158p.

BRASIL. Ministério da Saúde. O que é coronavírus? (COVID-19). 2020. Disponível em: https://coronavirus.saude.gov.br. Acesso em: 26 mar. 2020. 
BRASIL. VIGITEL 2018 - Vigilância dos Fatores de Risco e Proteção Para Doenças Crônicas por Inquérito Telefônico. Ministério da Saúde. Brasília, DF: MS, 2019. 131p.

CARR, Anitra C.; MAGGINI, Silvia. Vitamin C and immune function. Nutrients, v.9, n.11, p. 1211, 2017.

CDC. Center For Disease Control And Prevention, 2020. Coronavirus Disease 2019. Disponível em: https://www.cdc.gov/coronavirus/2019. Acesso em: 27 de mar de 2020.

CFN. Conselho Federal de Nutricionistas. Nota Oficial: Orientações à população e para os nutricionistas sobre o novo coronavírus. 2020. Disponível em: https://bit.ly/2QNVSo5. Acesso em: 25 mar. 2020.

FINAMORE, A; ROSELLI, M; DONINI, L.M; BRASILI, E; RAMI, R; CARNEVALI, P; MISTURA, L; PINTO, A; GIUSTI, AM; MENGHERI, E. Supplementation with Bifidobacterium longum Bar33 and Lactobacillus helveticus Bar13 mixture improves immunity in elderly humans (over 75 years) and aged mice. Nutrition, v. 63, p. 184-192, 2019.

GASMI, A; NOOR, S; TIPPAIROTE, T; DADAR, M; MENZEL, A; BJORKLUND, G. Individual risk management strategy and potential therapeutic options for the COVID-19 pandemic. Clinical Immunology, p. 108409, 2020. Disponível em: https://bit.ly/2KegNwG. Acesso em: 17 abr. 2020.

HARYANTO, B; SUKSMASARI, T; WINTERGERST, E; MAGGINI, S. Multivitamin supplementation supports immune function and ameliorates conditions triggered by reduced air quality. Vitam. Miner. 2015. Disponível em: https://bit.ly/2ROSsCa. Acesso em: 17 abr. 2020.

KHAN, S. U; KHAN, M. U; RIAZ,H; VALAVOOR, S; ZHAO, D; VAUGHAN, L; OKUNRINTEMI, V; RIAZ, IB; KHAN, MS; KALUSKI, E; MURAD, MH; BLAHA, MJ; GUALLAR, E. Effects of nutritional supplements and dietary interventions on cardiovascular outcomes: an umbrella review and evidence map. Annals of internal medicine, v. 171, n. 3, p. 190-198, 2019.

LASSELIN, Julie; ALVAREZ-SALAS, Elena; GRIGOLEIT, Jan-Sebastian. Well-being and immune response: a multi-system perspective. Current opinion in pharmacology, v. 29, p. 34-41, 2016.
LÓPEZ PLAZA, Bricia; BERMEJO LÓPEZ, Laura María. Nutrición y trastornos del sistema inmune. Nutrición Hospitalaria, v. 34, p. 68-71, 2017.

MAGGINI, S; PIERRE, A; CALDER, P.C. Immune function and micronutrient requirements change over the life course. Nutrients, v. 10, n. 10, p. 1531, 2018.

OPAS. Organização Pan-Americana da Saúde. Folha informativa - COVID-19. 2020. Disponível em: https://bit.ly/2UDjL2N. Acesso em: 26 mar. 2020.

PERBELIN, A.S; SILVA, C.V; MELLO, E.V.S.L; SCHNEIDER, L.C.L. The role of microbiota as allied in the immune system. Arquivos do MUDI, v. 23, n. 3, p. 345-358, 2019.

PROMPETCHARA, E; KETLOY, C; PALAGA, T. Immune responses in COVID-19 and potential vaccines: Lessons learned from SARS and MERS epidemic. Asian Pac J Allergy Immunol, 2020.

Disponível em: https://bit.ly/3biVJ4k. Acesso em: 26 mar. 2020.

UNIVERSIDADE ESTADUAL DE CAMPINAS. Tabela brasileira de composição de alimentosTACO. 161p. $4^{\circ}$ ed. 2011.

WANG, B; LI, R; LU, Z; HUANG, Y. Does comorbidity increase the risk of patients with COVID-19: evidence from meta-analysis. Aging, v. 12, 2020. Disponível em: https://bit.ly/2XMYSFG. Acesso em: 18 abr. 2020.

WATKINS, John. Preventing a covid-19 pandemic. $2020 . \quad$ Disponível em: https://www.bmj.com/content/368/bmj.m810. Acesso em: 18 abr. 2020.

WHO. World Healthy Organization (Switzerland). Healthy Diet. Genebra, 2018. 6p. (WHO Technical Report Series, 394).

ZHANG, L; LIU, Y. Potential interventions for novel coronavirus in China: A systematic review. Journal of medical virology, 2020. Disponível em: https://bit.ly/34LlJmk. Acesso em: 17 abr. 2020.

ZHOU, Fei et al. Clinical course and risk factors for mortality of adult inpatients with COVID-19 in Wuhan, China: a retrospective cohort study. The Lancet, 2020. Disponível em: https://bit.ly/2xFKYKK. Acesso em: 18 abr. 2020. 